\title{
Estudio comparativo de incidencia de bacteriemias relacionadas con el catéter de hemodiálisis: bioconectores frente a conexión directa
}

José Luis Cobo Sánchez ${ }^{1}$ - Rosa Ana Sainz Alonso² - $M^{2}$ Yolanda Vicente Jiménez ${ }^{2}$ - Hortensia Cepa García ${ }^{2}$ - Raquel Pelayo Alonso ${ }^{2}$ - Raquel Menezo Viadero² - Emilio Ibarguren Rodríguez ${ }^{2}$ - Ana Isabel Pérez Garmilla ${ }^{2}$ - Marina Rojo Tordable ${ }^{2}$ - Ana Begines Ramírez ${ }^{2}$ - $M^{\mathrm{a}}$ Teresa Sola García $^{2}$ - Rosa Alonso Nates ${ }^{2}$

${ }^{1}$ Máster en Ciencias de la Enfermería

${ }^{2}$ Diplomado en Enfermería

Servicio de Nefrología. Hospital Universitario Marqués de Valdecilla. Santander

\section{Resumen}

Objetivo: Comparar la tasa de bacteriemias relacionadas con el catéter de hemodiálisis entre el uso de bioconectores y la conexión directa.

Metodología: Estudio retrospectivo comparativo transversal durante un periodo de 2 años consecutivos ( 1 año con conexión directa - Febrero 2009 a Febrero 2010- y 1 año utilizando bioconectores Tego ${ }^{\circledR}$-Marzo 2010 a Marzo de 2011- ) en los pacientes en hemodiálisis de nuestra unidad que portaban un catéter como acceso vascular permanente. El protocolo de conexión y desconexión de los catéteres durante el periodo de estudio fue el mismo, a excepción de la solución de desinfección empleada: povidona iodada en la conexión directa y clorhexidina al $2 \%$ usando bioconectores. Se definió como bacteriemia relacionada con el catéter la aparición de fiebre junto con un hemocultivo positivo, en ausencia de otro foco de infección.

Dirección para la correspondencia: José Luis Cobo Sánchez Servicio de Nefrología

Hospital Universitario Marqués de Valdecilla Avda. Valdecilla s/n

39008 Santander. Cantabria

e-mail: jlcobosanchez@gmail.com
Resultados: Se estudiaron 69 pacientes: 36 durante el periodo de conexión directa y 33 durante el periodo del uso de bioconectores. Seis pacientes fueron estudiados durante ambos periodos. La tasa de bacteriemia durante el periodo de conexión directa fue de 24,6 bacteriemias/1000 catéteres-día ( 9 bacteriemias), mientras que durante el periodo de uso de bioconectores fue de 5,47 bacteriemias/1000 catéteres-día ( 2 bacteriemias), $(p=0,036)$. El microorganismo causal más común durante el periodo de conexión directa fue el Staphyloccocus aureus (3 pacientes) junto con el Staphyloccocus coagulasa negativo (3 pacientes).

Conclusiones: El uso de bioconectores disminuye de forma significativa la tasa de bacteriemias relacionadas con el catéter de hemodiálisis frente a la conexión directa.

\section{PALABRAS CLAVE:}

- HEMODIÁLISIS

- CATÉTERES

- INFECCIONES RELACIONADAS

CON CATÉTERES

- BACTERIEMIA

- BIOCONECTOR

- ATENCIÓN DE ENFERMERÍA 
Comparative study of the incidence of bacteraemia related to the haemodialysis catheter: bioconnectrs versus direct connection

\section{Abstract}

Objective: To compare hemodialysis catheterrelated bacteremia rate between bioconectors use and direct connection.

Methodology: Comparative, retrospective and cross-sectional study over 2 consecutive years (one year with direct connection - 2009 February to 2010 February - and 1 year using bioconectors Tego ${ }^{\circledR}-2010$ March to 2011 March-) in hemodialysis patients in our unit with a permanent catheter as vascular access. Catheters connection and disconnection protocol was the same during the study period, except for the disinfecting solution used: iodine povidone was using in direct connection, and $2 \%$ chlorhexidine was using with bioconectors. Catheterrelated bacteremia was defined as onset of fever with a positive blood culture in the absence of another infection source.

Results: 69 patients were studied: 36 during direct connection period and 33 during use bioconectors period. Six patients were studied during both periods. Bacteremia rate during direct connection period was 24.6/1000 catheters-day ( 9 bacteremia), while during the using bioconectors period, bacteremia rate was $5.47 / 1000$ catheters-day ( 2 bacteremia) $(p=0.036)$. Staphylococcus aureus ( 3 patients) and staphylococcus coagulase negative ( 3 patients) were the most common infectious agents during direct connection period.

Conclusions: Use of bioconectors significantly decreases the rate of bacteremia catheter-related in hemodialysis patients versus direct connection.

\section{KEY WORDS:}
- HEMODIALYSIS
- CATHETERS
- CATHETER-RELATED INFECTION
- BACTEREMIA
- LUER-LOCK BIOCONECTOR
- NURSING CARE

\section{Introducción}

El uso de catéteres venosos centrales (CVC) como acceso vascular (AV) permanente para los pacientes en hemodiálisis (HD) es una tendencia creciente a nivel global. Esta tendencia puede atribuirse a la remisión tardía a los servicios de Nefrología, el retraso en la formación del AV y a una mayor tasa de vasculopatías y diabetes, lo que resulta en un árbol vascular inadecuado para la formación de la fístula ${ }^{1,3}$.

El tipo de AV para HD puede contribuir de forma independiente a la mortalidad del paciente. Se ha reportado un aumento del riesgo de muerte siendo portador de un catéter de una a tres veces mayor en comparación con ser portador de una fístula ${ }^{4,6}$. Aunque no existen estadísticas específicas, las guías KDOQI argumentan que las complicaciones infecciosas asociadas con el uso de catéteres en los pacientes sometidos a HD contribuyen a que la infección sea la segunda causa principal de muerte en los pacientes con insuficiencia renal crónica ${ }^{7}$. La literatura refiere que la incidencia de los episodios de bacteriemia relacionados con el CVC de HD varían de 1,6 a 6,18/1000 catéteres-día ${ }^{8,9}$.

Por lo tanto, el cuidado del CVC debe ser una preocupación fundamental para la enfermería nefrológica, y la prevención de las infecciones relacionadas con el mismo, el objetivo principal durante el abordaje del CVC. En este sentido, además de la higiene de manos, el uso de mascarillas y la manipulación de forma estéril, existen productos en el mercado para prevenir las infecciones como soluciones de sellado con antibióticos y bioconectores tipo luer lock ${ }^{10}$.

El objetivo de los bioconectores es crear una luz sin obstáculos en la posición abierta, con una resistencia equivalente a la obtenida cuando se conecta la línea directamente al catéter. El bloqueo del conector está formado por una capa de silicona para evitar la hemólisis. De esta manera se crea un sellado continuo, que evita la contaminación microbiológica. Dos recientes estudios han demostrado que su uso no influye en los flujos y resistencias frente a la conexión directa ${ }^{11,12}$.

El objetivo del presente estudio fue comparar la tasa de bacteriemias relacionadas con el CVC de HD entre el uso de bioconectores y la conexión directa. 


\section{Metodología}

Se llevó a cabo un estudio retrospectivo comparativo transversal, durante un periodo de 2 años consecutivos (1 año sin usar bioconectores - Febrero 2009 a Febrero 2010- y 1 año utilizando bioconectores Tego ${ }^{\circledR}$ -Marzo 2010 a Marzo de 2011-) en los pacientes en HD crónica de nuestra unidad que disponían de un CVC como AV permanente.

El protocolo de conexión y desconexión de los catéteres durante el periodo de estudio fue el mismo: uso de mascarillas por parte del paciente y de la enfermera, lavado de manos previo a la manipulación, manipulación del catéter bajo máximas medidas de asepsia creando un campo estéril a partir de los guantes estériles para la preparación del material necesario tanto para la conexión como para la desconexión, y cubierta del catéter durante la sesión de HD con paño estéril.
La única variación, además del uso de bioconectores, fue la solución de desinfección empleada: durante el periodo de conexión directa se utilizó povidona iodada y durante el periodo del uso de bioconectores se utilizó clorhexidina al $2 \%$, ya que la povidona iodada daña la silicona.

En ambos periodos se cubrieron las conexiones entre las líneas de sangre y el catéter con gasas estériles impregnadas en la solución desinfectante utilizada en cada periodo, durante toda la sesión.

Los bioconectores se cambiaron cada 7 días, el primer día de la semana que el paciente se dializaba. Durante el periodo de conexión directa las luces del CVC se cerraban con tapones convencionales, que se cambiaban en cada sesión de HD. Durante ambos periodos, la solución de sellado de los catéteres fue heparina al $5 \%$, utilizando sólo uroquinasa $5000 \mathrm{UI} / \mathrm{ml}$ de manera puntual si existían problemas de flujo.

\begin{tabular}{|c|c|c|c|}
\hline & Conexión directa & Uso de bioconectores & $\mathbf{p}$ \\
\hline Edad (años) & $67 \pm 13$ & $67 \pm 11$ & 0,871 \\
\hline \multicolumn{4}{|l|}{ Sexo (N (\%)) } \\
\hline Mujeres & $12(33,3 \%)$ & $6(18,8 \%)$ & \multirow[t]{2}{*}{0,139} \\
\hline Hombres & $24(66,7 \%)$ & $26(81,2 \%)$ & \\
\hline \multicolumn{4}{|c|}{ Etiología insuficiencia renal (N (\%)) } \\
\hline Sistémica & $9(25 \%)$ & $9(28,1 \%)$ & \multirow[t]{7}{*}{0,870} \\
\hline Vascular & $10(27,8 \%)$ & $12(37,5 \%)$ & \\
\hline Congénita & $3(8,3 \%)$ & $3(9,4 \%)$ & \\
\hline Glomerular & $7(19,4 \%)$ & $5(15,6 \%)$ & \\
\hline Intersticial & $3(8,3 \%)$ & $2(6,3 \%)$ & \\
\hline Desconocida & $3(8,3 \%)$ & $1(3,1 \%)$ & \\
\hline Otras & $1(2,8 \%)$ & - & \\
\hline \multicolumn{4}{|l|}{ Bacteriemia (N (\%)) } \\
\hline Si & $9(25 \%)$ & $2(6,3 \%)$ & \multirow[t]{2}{*}{0,036} \\
\hline No & $27(75 \%)$ & $30(93,7 \%)$ & \\
\hline \multicolumn{4}{|l|}{ Tipo de catéter ( $\mathrm{N}(\%)$ ) } \\
\hline Tessio & $32(88,9 \%)$ & $32(100 \%)$ & \multirow[t]{3}{*}{0,062} \\
\hline Percath & $1(2,8 \%)$ & - & \\
\hline Palindrome & $3(8,3 \%)$ & - & \\
\hline \multicolumn{4}{|l|}{ Localización catéter ( $\mathrm{N}(\%)$ ) } \\
\hline Yugular & $35(97 \%)$ & $30(93,8 \%)$ & \multirow[t]{3}{*}{0,073} \\
\hline Un ramal en cada yugular & - & $1(3,1 \%)$ & \\
\hline Femoral & $1(3 \%)$ & $1(3,1 \%)$ & \\
\hline
\end{tabular}

Tabla 1. Características clínicas y sociodemográficas, incluida presencia de bacteriemia, durante los 2 periodos de estudio (conexión directa y uso de bioconectores), junto con la significación estadística para cada parámetro 
Se definió como bacteriemia relacionada con el CVC la aparición de fiebre junto con un hemocultivo positivo, en ausencia de otro foco de infección. La tasa de incidencia de bacteriemia se calculó como el $\mathrm{N}^{\circ}$ bacteriemias / días de seguimiento x 1000 .

Se recogieron datos clínicos y sociodemográficos de la historia clínica del paciente: edad, sexo, etiología de la insuficiencia renal, tipo de catéter, localización, presencia de bacteriemia y agente infeccioso.

Los datos recogidos fueron analizados mediante el paquete estadístico SPSS versión 15.0 para Windows. Se realizó un análisis descriptivo de los resultados obtenidos. Para la inferencia estadística se utilizaron los test Chi-cuadrado y T de Student. Los resultados se consideraron significativos si el nivel crítico observado era inferior al $5 \%(p<0,05)$.

\section{Resultados}

Se estudiaron 69 pacientes: 36 durante el periodo de conexión directa y 33 durante el periodo del uso de bioconectores. Seis pacientes fueron estudiados durante ambos periodos. Las características clínicas y sociodemográficas de los pacientes durante ambos periodos de estudio se muestran en la tabla 1 . No hubo diferencias estadísticamente significativas entre las características clínicas de los pacientes estudiados durante ambos periodos, excepto en el tipo catéter, que durante el periodo de uso de bioconectores fueron todos tipo Tessio.

En la tabla 2 se muestran las características de los pacientes que presentaron bacteriemias durante cada periodo de estudio, junto con el agente microbiano causante. Durante el periodo de conexión directa, un

\begin{tabular}{|c|c|c|c|}
\hline \multicolumn{4}{|c|}{ Conexión directa } \\
\hline & Filiación paciente & Filiación catéter & Microorganismo bacteriemia \\
\hline Paciente 1 & $\begin{array}{l}\text { Hombre, } 75 \text { años. } \\
\text { Nefritis Tubulo-Intersticial. }\end{array}$ & $\begin{array}{l}\text { Tessio. } \\
\text { Inserción en yugular derecha. }\end{array}$ & Staphyloccocus aureus \\
\hline Paciente 2 & $\begin{array}{l}\text { Hombre, } 79 \text { años. } \\
\text { Nefropatía intersticial. }\end{array}$ & $\begin{array}{l}\text { Tessio. } \\
\text { Inserción en yugular derecha. }\end{array}$ & Staphyloccocus coagulasa negativo \\
\hline Paciente 3 & $\begin{array}{l}\text { Mujer, } 83 \text { años. } \\
\text { Nefroangioesclerosis. }\end{array}$ & $\begin{array}{l}\text { Tessio. } \\
\text { Inserción en yugular izquierda. }\end{array}$ & Staphyloccocus coagulasa negativo \\
\hline Paciente 4 & $\begin{array}{l}\text { Hombre, } 65 \text { años. } \\
\text { Nefroangioesclerosis. }\end{array}$ & $\begin{array}{l}\text { Tessio. } \\
\text { Inserción en femoral izquierda. }\end{array}$ & $\begin{array}{l}\text { Staphyloccocus epidermidis } \\
\text { Micrococcus }\end{array}$ \\
\hline Paciente 5 & $\begin{array}{l}\text { Mujer, } 49 \text { años. } \\
\text { Glomerulonefritis crónica. }\end{array}$ & $\begin{array}{l}\text { Tessio. } \\
\text { Inserción en yugular derecha. }\end{array}$ & Staphyloccocus epidermidis \\
\hline Paciente 6 & $\begin{array}{l}\text { Mujer, } 58 \text { años. } \\
\text { Glomerulonefritis. }\end{array}$ & $\begin{array}{l}\text { Tessio. } \\
\text { Inserción en yugular izquierda. }\end{array}$ & Staphyloccocus aureus \\
\hline Paciente 7 & $\begin{array}{l}\text { Hombre, } 67 \text { años. } \\
\text { Nefropatía diabética. }\end{array}$ & $\begin{array}{l}\text { Tessio. } \\
\text { Inserción en yugular derecha. }\end{array}$ & Staphyloccocus aureus \\
\hline Paciente 8 & $\begin{array}{l}\text { Hombre, } 57 \text { años. } \\
\text { Glomerulonefritis. }\end{array}$ & $\begin{array}{l}\text { Tessio. } \\
\text { Inserción en yugular derecha }\end{array}$ & Staphyloccocus coagulasa negativo \\
\hline \multicolumn{4}{|c|}{ Uso de bioconectores } \\
\hline & Filiación paciente & Filiación catéter & Microorganismo bacteriemia \\
\hline Paciente 1 & $\begin{array}{l}\text { Hombre, } 52 \text { años. } \\
\text { Insuficiencia renal secundaria a } \\
\text { mieloma múltiple. }\end{array}$ & $\begin{array}{l}\text { Tessio. } \\
\text { Inserción en yugular derecha. }\end{array}$ & Staphyloccocus coagulasa negativo \\
\hline Paciente 2 & $\begin{array}{l}\text { Hombre, } 81 \text { años. } \\
\text { Poliquistosis hepatorenal. }\end{array}$ & $\begin{array}{l}\text { Tessio. } \\
\text { Un ramal en cada yugular. }\end{array}$ & Staphyloccocus aureus \\
\hline
\end{tabular}

Tabla 2. Características clínicas de los pacientes que presentaron bacteriemias relacionadas con el catéter de hemodiálisis durante los 2 periodos de estudio 
paciente presentó 2 bacteriemias relacionadas con el catéter (paciente 4 de la tabla 2).

La tasa de bacteriemia durante el periodo de conexión directa fue de 24,6 bacteriemias/ 1000 catéteres-día, mientras que durante el periodo de uso de bioconectores fue de 5,47 bacteriemias/ 1000 catéteres-día $(p=0,036)$.

\section{Discusión}

Los datos de nuestro estudio muestran una disminución drástica en la tasa de bacteriemias durante el periodo de uso de bioconectores comparado con la conexión directa de las redes sanguíneas a la luz del catéter. Es conocido que la contaminación de la boca del catéter contribuye sustancialmente a la colonización intraluminal de los catéteres de larga duración. Las múltiples manipulaciones que sufre el catéter de HD durante la misma, contribuyen a la contaminación de la luz del mismo. El bioconector, al crear un sellado automático de forma continua, creando un sistema cerrado mecánica y microbiológicamente, evita la contaminación durante la conexión y desconexión de las redes.

Otros estudios refieren resultados dispares en el uso de los bioconectores. Bouza y cols estudiaron la tasa de bacteriemias utilizando bioconectores frente al uso de tapones convencionales en CVC de pacientes ingresados en unidades de cuidados intensivos. Entre estos catéteres, estudiaron catéteres de HD, observando una tasa de bacteriemias de 3,78 por cada 1000 catéteres/día usando bioconectores frente a 5,89 por cada 1000 catéteres/día usando los tapones convencionales $(p=0,4)^{13}$. Bort y cols evaluaron a 12 pacientes en HD, comparando el conector Tego ${ }^{\circledR}$ frente al uso de tapones convencionales, durante un periodo de 2 meses (un mes con cada tipo), estudiando las presiones del circuito, la eficacia dialítica y la tasa de infecciones. No encontraron bacteriemias relacionadas con el catéter durante ese periodo de tiempo, a pesar de presentar cultivos positivos en el $44 \%$ de los orificios de salida ${ }^{11}$. McAfee y cols compararon la tasa de bacteriemias en 15 pacientes pediátricos en HD utilizando bioconectores Tego ${ }^{\circledR}$ con la conexión directa al catéter, observando una tasa de 7,8 infecciones por cada 1000 pacientesdía con la conexión directa y de 3,65 infecciones por cada 1000 pacientes-día utilizando bioconectores. La incidencia de bacteriemias reportada por este estudio fue de 0,47 (IC 95\%: 0,23-0,96) con el uso de bioconector, menos de la mitad que utilizando la conexión directa $(p=0,04)^{14}$.

El otro factor que pudiese influir en la baja tasa de bacteriemias, además del uso de bioconectores, fue la solución de desinfección empleada. La clorhexidina se ha revelado en los últimos años como un buen agente antimicrobiano. Martín y cols. evaluaron la incidencia de bacteriemias utilizando un nuevo protocolo de conexión y desconexión de los catéteres de HD en el que utilizaron clorhexidina como solución de desinfección de los ramales, durante un periodo de 1 año, presentando una incidencia de bacteriemias de 1,8 bacteriemias/ 1.000 catéteres-día ${ }^{15}$. Lo que parece claro es que la combinación de ambos, bioconectores y clorhexidina, disminuye de forma significativa la tasa de bacteriemia relacionada con el CVC.

La manipulación de los accesos vasculares de los pacientes en HD, conlleva una gran responsabilidad, ya que las posibles complicaciones derivadas de la misma dan lugar tanto a la disminución en la seguridad y supervivencia del paciente como a la del propio acceso vascular. El lavado de manos, junto con el uso de mascarillas y una manipulación lo más estéril posible, son la base del manejo de los catéteres de HD. Las enfermeras nefrológicas debemos reflexionar sobre la importancia que tienen los conocimientos acerca de los aspectos específicos que tiene nuestra especialidad y su repercusión sobre la salud de los pacientes y el coste que conlleva la aparición de complicaciones derivadas de la falta de conocimientos. Aunque no ha sido objeto de nuestra investigación, está claro que el factor más importante en la prevención de las bacteriemias relacionadas con los CVC son los conocimientos de los profesionales que manejan los mismos, por lo que la formación reglada en nuestra especialidad y la estabilidad en las plantillas, contribuyen a un menor número de efectos secundarios derivados del tratamiento sustitutivo renal en nuestros pacientes.

\section{Conclusiones}

El uso de bioconectores disminuye de forma significativa la tasa de bacteriemias relacionadas con el CVC de HD frente a la conexión directa. La sinergia de los 
bioconectores junto con el uso de clorhexidina aumenta la protección frente a las bacteriemias.

Recibido: Septiembre 2011

Revisado: Septiembre 2011

Modificado: Octubre 2011

Aceptado: Octubre 2011

\section{Bibliografía}

1. Butterly DW, Schwab SJ. Catheter access for hemodialysis: an overview. Semin Dial 2001; 14(6): 411-5.

2. Letourneau I, Ouimet $D$, Dumont $M$, Pichette $V$, Leblanc M. Renal replacement in end-stage renal disease patients over 75 years old. Am J Nephrol 2003; 23(2): 71-7.

3. Mendelssohn DC, Ethier J, Elder SJ, Saran R, Port FK, Pisoni RL. Haemodialysis vascular access problems in Canada: results from the dialysis outcomes and practice patterns study (DOPPS II). Nephrol Dial Transplant 2006; 21(3): 721-8.

4. Dhingra RK, Young EW, Hulbert-Shearon TE, Leavey SF, Port FK. Type of vascular access and mortality in US hemodialysis patients. Kidney Int 2001; 60(4): 1443-51.

5. Pastan S, Soucie JM, McClellan WM. Vascular access and increased risk of death among hemodialysis patients. Kidney Int 2002; 62(2): 620-6.

6. Polkinghorne KR, McDonald SP, Atkins RC, Kerr PG. Vascular access and all cause mortality: a propensity score analysis. J Am Soc Nephrol 2004; 15(2): 477-86.

7. National Kidney Foundation. KDOQI Clinical Practice Guidelines and Clinical Practice Recommendations for 2006 Updates: Hemodialysis Adequacy, Peritoneal Dialysis Adequacy and Vascular Access. Am J Kidney Dis 2006; 48 (suppl 1):S1-S322.

8. Saeed Abdulrahman I, Al-Mueilo SH, Bokhary HL, Ladipo G0, Al-Rubaish A. A prospective study of hemodialysis access related bacterial infections. $J$ Infect Chemother 2002; 8(3): 242-6.

9. Weijmere MC, Vervloet MG, ter Wee PM. Compared to tunnelled cuffed haemodialysis catheters, temporary untunnelled catheters are associated with more complications already within 2 weeks of use. Nephrol Dial Transplant 2004; 19(3): 670-7.

10. Dinwiddie LC, Bhola C. Hemodialysis catheter care: Current recommendations for nursing practice in North America. Nephrol Nurs J 2010; 37(5): 507521.

11. Bort Castelló J, Salvador Lengua C, Mallol Domínguez A, Carratalá Chacón J, Cerrillo García V, Folch Morro MJ y cols. Evaluación de un nuevo dispositivo de conexión para los catéteres de hemodiálisis. Rev Soc Esp Enferm Nefrol 2010; 13 (3): 168-172.

12. Cobo Sánchez JL, Incera Setién $M E$, Vicente Jiménez MY, Sainz Alonso R, Sáenz de Buruaga Perea A, Díez Rumayor M y cols. Estudio comparativo de flujos y resistencias de catéteres para hemodiálisis usando bioconectores luer-lock. Rev Soc Esp Enferm Nefrol 2010; 13 (1): 23-28.

13. Bouza $E$, Muñoz $P$, López Rodríguez J, Pérez MJ, Rincón P, Martín Rabadán P y cols. A needleless closed system device (CLAVE) protects from intravascular catheter tip and hub colonization: A prospective randomized study. J Hosp Infect 2003; 54: 279-287.

14. McAfee N, Seidel K, Watkins S, Flynn JT. A continuous quality improvement project to decrease hemodialysis catheter infections in pediatric patients: use of a closed luer-lock access cap. Nephrol Nurs J 2010; 37(5):541-4.

15. Martín Chacón E, Martín Álvarez MC, Sánchez Calzado M, Banegas Sultán V, Gil del Reino 0, Menayo Gómez $\mathrm{J}$ y cols. Incidencia de bacteriemia en pacientes portadores de catéter permanente tunelizado para hemodiálisis. Rev Soc Esp Enferm Nefrol 2008; 11 (4): 277-281. 